Check for updates

Cite this: RSC Adv., 2017, 7, 31310

Received 18th March 2017 Accepted 12th June 2017

DOI: 10.1039/c7ra03193a

rsc.li/rsc-advances

\section{Fabrication of ascorbyl palmitate loaded poly(caprolactone)/silver nanoparticle embedded poly(vinyl alcohol) hybrid nanofibre mats as active wound dressings via dual-spinneret electrospinning $\dagger$}

\begin{abstract}
L. Du, (DD ${ }^{\text {ab }}$ H. Z. Xu, ${ }^{c}$ T. Li, ${ }^{a}$ Y. Zhang ${ }^{a b}$ and F. Y. Zou*ab
In this study, a good combination consisting of poly(vinyl alcohol) (PVA) incorporated with monodisperse silver nanoparticles (AgNPs) and poly(caprolactone) (PCL) loaded with ascorbyl palmitate (AP) was introduced for wound healing. The hybrid nanofibrous mats were analysed by ultraviolet-visible spectroscopy (UV-Vis), scanning electron microscopy (SEM), transmission electron microscopy (TEM), $X$-ray diffraction (XRD), X-ray photoelectron spectroscopy (XPS), and Fourier transform infrared spectroscopy (FTIR). UV-vis and TEM revealed the presence and morphology of well-dispersed AgNPs obtained by an in situ reduction approach in the PVA nanofibres after electrospinning. XPS and XRD results demonstrated that the AgNPs and AP were physically mixed into the PVA and PCL, respectively. Successful preparation of bicomponent polymer matrices (PVA and PCL) in the hybrid mat was confirmed by FTIR and XRD. To evaluate the cytocompatibility of the hybrid nanofibre mats, NIH-3T3 fibroblast cells were seeded on the matrix. Results showed that the incorporation of AP could weaken the toxic effects of AgNPs on cell proliferation. The antibacterial effects on Escherichia coli (E. coli) and Staphylococcus aureus (S. aureus), and the animal wound healing capabilities were also investigated. The hybrid nanofibres mats showed a high antibacterial activity against $E$. coli and S. aureus. The wound healing test and histological evaluation also revealed that the AP loaded PCL/AgNP embedded PVA hybrid nanofibre mats will have potential as a wound dressing for future therapeutic biomedical applications.
\end{abstract}

\section{Introduction}

The skin is the largest organ in the bodies of vertebrates, and it is the outermost barrier to protect the inner organs from microbial pathogens, and mechanical and chemical damage. Skin lesions can occur due to burns, acute traumas or chemical agents. ${ }^{1}$ Wound healing is a complex process involving homeostasis, inflammation, cellular proliferation/migration, and tissue remodeling. ${ }^{2}$ The wound self-healing process faces a number of anomalies such as microorganism infection and tissue disruption. ${ }^{3}$ Wound dressings are essential for the prevention of infection and shorten the skin recovery period.

${ }^{a}$ School of Fashion Design \& Engineering, Zhejiang Sci-Tech University, Hangzhou 310018, China. E-mail: zfy166@zstu.edu.cn; Fax: +86-571-86843249

${ }^{b}$ Zhejiang Provincial Research Center of Clothing Engineering Technology, Zhejiang Sci-Tech University, Hangzhou 310018, China

${ }^{c}$ Department of Bio-based Materials Science, Kyoto Institute of Technology, Kyoto 6068585, Japan

$\dagger$ Electronic supplementary information (ESI) available. See DOI: 10.1039/c7ra03193a
Various efforts have been made in finding new wound dressings with good properties to provide surface protection, bacteriostatic controlling activities, and moisture maintenance. ${ }^{\mathbf{4 - 6}}$ Electrospun nanofibres show great promise for the development of wound dressings due to their predominant properties such as high specific surface area, high porosity, and flexibility in surface functionalities. ${ }^{7-11}$ throughout the whole wound healing process since it requires not only protecting from invading microbes, but also participating in the tissue repairing. ${ }^{12}$ To prevent bacterial colonization and infection, antimicrobial agents should be considered embedding into the electrospun nanofibres. Silver nanoparticles (AgNPs) have an attractive anti-microbial activity which can be attributed to a strong interaction with bacterial cells. ${ }^{13}$ It is also well known that broad-acting biocidal activity of AgNPs is closely related to their size, shape, and surface properties. ${ }^{14}$ However, the AgNPs trend to aggregate due to the high surface energy. Thus, AgNPs incorporated within biodegradable polymers to maintain stability, and antibacterial activity is of the utmost necessity. ${ }^{15-18}$ There are also many methods to
The inflammatory phase is the most important stage 
synthesize AgNPs. Youk et al. ${ }^{19}$ reduced $\mathrm{Ag}^{+}$ions using the annealing process at different temperatures and time. Lin et $a .^{20}$ used ultraviolet light irradiation to reduce $\mathrm{AgNO}_{3}$. Selvam et al. ${ }^{21}$ used dimethylformamide (DMF) etc. chemical reductants to synthesize the AgNPs. However, greener reductants assisted biomimetic synthesis of AgNPs have received a great deal of attention. Shukla et $a .^{22}$ and Du et al. ${ }^{23-25}$ applied black pepper, and tea polyphenols respectively acted as reductants for the formation of AgNPs. In the wound healing process, green reducing agents need to be employed to reduce the generation of substances hazardous to human health.

Wound healing is also an interactively dynamic process and intercellular stimulating response. The adding of vitamins, minerals, growth factors and other wound healing accelerators will the promote rapid healing. ${ }^{26}$ Ascorbyl palmitate (AP) is a fatsoluble derivative of ascorbic acid (vitamin C). AP is not only more stable than ascorbic acid, but also has the equal antioxidant activity to the vitamin $\mathrm{C}^{27,28}$ which could promote tissue engraftment and specifically increase type I and type III procollagen in human skin fibroblasts. ${ }^{29,30}$

The ideal wound dressing should be capable of treating bacterial infection and encouraging the healing process. ${ }^{31}$ Different strategies are currently used by researchers to develop hybrid electrospun nanofibrous mat wound dressings consisting of two or more components since those types of dressings present synergistic effects of individual component to improve the overall properties of the wound dressings. ${ }^{32-35}$ Herein, the present study aims at preparing and characterizing hybrid nanofibrous mat wound dressings with four components: poly(vinyl alcohol) (PVA), poly(caprolactone) (PCL), silver nanoparticles (AgNPs), and ascorbyl palmitate (AP), respectively. In particular, the PVA and PCL are the two quintessential polyesters, whose excellent biocompatibilities and biodegradable nature allow their utilization as tissue scaffolds, artificial organs and wound dressing. ${ }^{36-40}$ PVA could keep moist environment to promote cell adhesion and proliferation because of its hydrophilicity. PCL maintains structural stability since it is a highly elastic and soft-and-hard-tissue-compatible bioresorbable material. ${ }^{41-43}$ The loading of AgNPs can endow antibacteria ability to the dressing, while AP can strengthen healpromoting activity.

\section{Materials and methods}

\subsection{Materials}

Poly(caprolactone) (PCL) pellets $M_{\mathrm{n}}=80000 \mathrm{~g} \mathrm{~mol}^{-1}$ was purchased from Sigma Aldrich, America. Poly(vinyl alcohol) (PVA) powders (99\% hydrolyzed, $M_{\mathrm{w}}=145000 \mathrm{~g} \mathrm{~mol}^{-1}$ ) and silver nitrate $\left(\mathrm{AgNO}_{3}\right.$, purity $\left.99.8 \%\right)$ were obtained from Aladdin Chemistry Co., Ltd, China. Tea polyphenols (TP, purity 98\%) was acquired from Shanghai Jinsui Bio-Technology Co., Ltd, China. Ascorbyl palmitate (AP, purity 97\%) was purchased from Tokyo Chemical Industry Co., Ltd., Japan. The reagent grade $N, N$-dimethylformamide (DMF) and dichloromethane (DCM) were obtained from Yongda Chemical Reagent Co., Ltd., China. Glutaraldehyde (GA, $25 \mathrm{v} / \mathrm{v}$ in water) was purchased from Kermel Chemical Reagent Co., Ltd, China. Nutrient agar was purchased from Hangzhou Baisi biotechnology Co., Ltd., China. Deionized water (DIW) with a resistance of $c a .18 \mathrm{M} \Omega \mathrm{cm}$ was used in the experiment. All the chemicals were used as received without further purification.

\subsection{Preparation of electrospun precursor solution}

PVA solution was prepared by fully dissolving with magnetic stirring $1.667 \mathrm{~g}$ polymer powder in the $13 \mathrm{~mL}$ DIW for $1.5 \mathrm{~h}$, at the temperature of $80{ }^{\circ} \mathrm{C}$. PVA solution (10 wt\%) was cooled down to room temperature. Then, $0.0334 \mathrm{~g} \mathrm{AgNO}_{3}$ (i.e. $2 \mathrm{wt} \%$ relative to the weight of PVA) was added in the PVA solution, and the mixture was kept uninterruptedly stirring at $65{ }^{\circ} \mathrm{C}$ for $0.5 \mathrm{~h}$. After that, the $0.02 \mathrm{~g}$ TP was dissolved in $2 \mathrm{~mL}$ DIW solution and dropwise added to the PVA/AgNO $\mathrm{AN}_{3}$ solution. PCL solution (12 wt\%) was prepared by dissolving $1.757 \mathrm{~g}$ PCL pellets in $10 \mathrm{~mL} \mathrm{DCM} / \mathrm{DMF} 9: 1 \mathrm{v} / \mathrm{v}$ solvent and $0.176 \mathrm{~g}$ AP was added in PCL solution by dissolving its powder in corresponding amount of $10 \mathrm{wt} \%$ with respect to PCL. The mixture was kept stirring for $3 \mathrm{~h}$ at room temperature to achieve a homogenous solution.

\subsection{Electrospinning of the hybrid polymer solutions}

To prepare the PCL/PVA hybrid nanofibrous mat containing AP and AgNPs, an electrospinning setup featuring two polymer solutions delivery systems was designed. The polymer solutions were contained in syringes located on both sides of the collector at an angle of $180^{\circ}$, as illustrated in Fig. 1. The two fluid flow rates were kept at $8 \mu \mathrm{L} \mathrm{min}{ }^{-1}$, and the distance between the needle tip and the collector were $12 \mathrm{~cm}$. The voltages used for AgNPs/PVA and PCL/AP were $12 \mathrm{kV}$ and $10 \mathrm{kV}$, respectively. The speed of the rolling cylinder was controlled at $200 \mathrm{rpm}$. The electrospinning of the polymer solution lasted for approximate $4 \mathrm{~h}$. For comparison, the PCL/PVA NFNs without AgNPs or AP were prepared similarly as the above procedure. Meanwhile, neat PCL and PVA nanofibre mats were also fabricated by conventional single nozzle electrospinning, using the same conditions above. All experiments were conducted at $22-25{ }^{\circ} \mathrm{C}$ with a relative humidity of $27-30 \%$. To obtain PVA nanofibres with water stability, the samples contained PVA were crosslinked in glutaraldehyde vapor (GA) in a vacuum chamber at $30{ }^{\circ} \mathrm{C}$ for $48 \mathrm{~h}$. The crosslinked nanofibrous mats were dried for

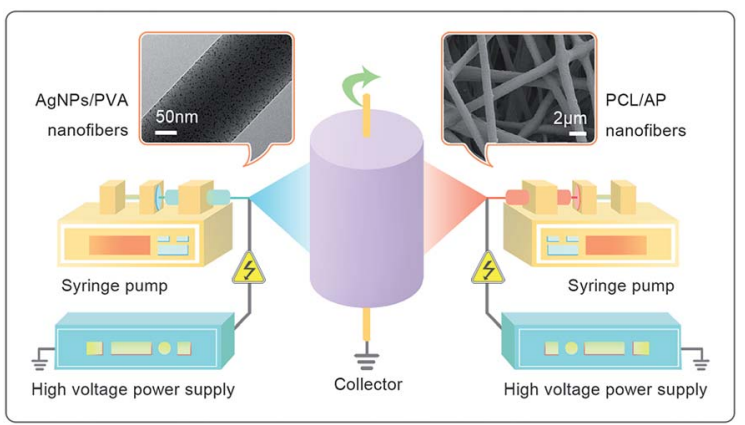

Fig. 1 Schematic illustration of the designed setup of the two-spinneret electrospinning apparatus. 
$24 \mathrm{~h}$ in the vacuum drier at room temperature to remove residual solvents.

\subsection{Ultraviolet-visible (UV-vis) spectrometer measurements}

UV-vis spectra were examined by a Lambda 35 UV-vis spectrophotometer (Perkin Elmer, USA) to confirm AgNPs formation. The DIW was used as the blank, and samples were taken in the by 6 time-points, i.e., $5 \mathrm{~min}, 15 \mathrm{~min}, 30 \mathrm{~min}, 45 \mathrm{~min}, 60 \mathrm{~min}$ and $90 \mathrm{~min}$, respectively. For each time point, $0.2 \mathrm{~mL}$ PVA solution with AgNPs was taken and diluted 10-fold. All the spectra were collected over a wavelength range of $200-800 \mathrm{~nm}$.

\subsection{Morphologies of the electrospun hybrid nanofibres}

The fibres morphologies were studied with Field Emission Scanning Electron Microscope (FE-SEM, Ultra 55, Zeiss, Germany). After being vacuum-coated with a thin layer of platinum at $40 \mathrm{~mA}$ for 40 seconds, the fibres were observed using the SEM at $3 \mathrm{kV}$. The diameters of the nanofibres were determined from the FE-SEM image using the Image Pro-Plus software (200 fibres of were randomly selected for the measurement). The Transmission Electron Microscopy (TEM) image acquired via a JSM2100 Transmission Electron Microscope (JEOL, Japan) was at an accelerating voltage of $200 \mathrm{kV}$. The size of the AgNPs was also measured using Image Pro-Plus software from the TEM images $(n=200)$.

\subsection{X-ray photoelectron spectroscopy}

The chemical composition changes of nanofibres were determined by X-ray photoelectron spectroscopy (XPS, K-Alpha, Thermo Scientific, America). The XPS spectrum was differentiated and fitted using XPS peak software. The binding energy (B.E) was corrected by setting the C 1 s peak (C-C) at $284.8 \mathrm{eV}$. The high-resolution survey was performed at spectral regions relating to carbon, oxygen, and silver.

\subsection{X-ray diffraction (XRD) analysis}

The crystalline structure of the samples was determined by $\mathrm{X}$ ray diffraction (XRD, D8 discover, Bruker, Germany) with $\mathrm{Cu}$ $\mathrm{K}_{\alpha}$ radiation $(\lambda=1.5406 \AA)$ in the range of $10-90^{\circ}$ at a scan speed of $3^{\circ} \min ^{-1}$.

\subsection{Fourier transform infrared spectroscopy}

All infrared spectra were acquired using a Thermo Nicolet 5700 Fourier transform infrared spectrometer. All spectra were recorded in the attenuated total reflectance Fourier transform infrared spectroscopy (ATR-FTIR) mode at a resolution of 4 $\mathrm{cm}^{-1}$ and 32 scans.

\subsection{Inhibition zone assay}

The antimicrobial activity of the composite mats was investigated against Gram-negative bacteria Escherichia coli (E. coli, ATCC 25922) and Gram-positive bacteria Staphylococcus aureus (S. aureus, ATCC 6538), using the disk diffusion method (ISO 20645: 2004). $100 \mu \mathrm{L} \mathrm{E}$. coli and $S$. aureus were cultured on Luria-Bertani (LB) agar plates used for the antibacterial activity tests. Alone PVA/PCL nanofibre was used as the control. The PVA/PCL containing AgNPs, AP, and AgNPs/AP were used as the test samples. The mats were cut into small circular pieces (12 $\mathrm{mm}$ ) and placed over the solidified agar gel. Then the plates were incubated for $18 \mathrm{~h}$ at $37^{\circ} \mathrm{C}$ in a bacteriological incubator. Subsequently, the zone of bacterial inhibition was monitored.

\subsection{Cytotoxicity of electrospun hybrid nanofibres}

Standard MTT assay was performed to evaluate the biocompatibility of four different nanofibres mats. The samples were pierced into $6 \mathrm{~mm}$ round disk to fit in a 96-well plate. A $100 \mu \mathrm{L}$ of mouse fibroblast cell line $(\mathrm{NIH}-3 \mathrm{T3})$ suspension containing was carefully seeded on top of sterilized nanofibre. After cell seeding for 1, 4, and 7 days in 96-well plate, the nanofibrous dressings with attached cells were incubated with $1 \mathrm{mg} \mathrm{mL}^{-1}$ thiazolyl blue (3-[4,5-dimethylthiazol-2-yl]-2,5-diphenyltetrazolium bromide, MTT) for $4 \mathrm{~h}$ at $37^{\circ} \mathrm{C}$. After removing the medium, $150 \mathrm{~mL}$ dimethyl sulfoxide (DMSO) was added to each well and shook for $10 \mathrm{~min}$, the absorbance of the supernatant at $490 \mathrm{~nm}$ was determined by a microplate reader (Elx800, Bio-Tek, America). A total of five replicate samples were performed on each group.

\subsection{In vivo wound healing study}

The in vivo animal study was performed in strict accordance with the NIH guidelines for the care and use of laboratory animals (NIH Publication No. 85-23 Rev. 1996) and was approved by the Institutional Animal Ethical Committee, Experimental Animal Centre of the Hangzhou Normal University. Twenty-five male rats weighing $(200 \pm 20 \mathrm{~g})$ between 4 and 6 weeks of age were used as the test animals. Rats were randomly divided into five groups based on the materials to be used to dress the skin wounds. A full thickness wound with a surface area of $1.5 \times 1.5 \mathrm{~cm}^{2}$ was cut from the dorsum of the rats. The wounds were covered with an equal size of electrospun nanofibres mats (i.e. neat PVA/PCL, PVA/PCL/AgNPs, PVA/PCL/ $\mathrm{AP}$, and PVA/PCL/AgNPs/AP). The rats with bare wound were kept covered using cotton gauze. After the dressing materials had been fixed, the rats were housed individually in cages under normal room temperature. The dressings were changed every 3 days after grafting. When changing dressings, the appearance of the wound was photographed. After day 18, the skin wound tissue of the rats were excised and fixed with $10 \%$ formalin. The specimens were stained with hematoxylin-eosin (H\&E) to perform histological observations.

\section{Results and discussion}

\subsection{Physicochemical characterization of AgNPs via UV-vis spectra}

The formation of silver nanoparticles was confirmed using UVvis spectra. The broad plasma resonance peak around $435 \mathrm{~nm}$ can be assigned to surface plasmon resonance (SPR) of AgNPs (see Fig. 2). The intensities of the SPR absorption of the AgNPs increased with the reaction time, indicating that more AgNPs were generated. The bright yellowish colour is observed after 


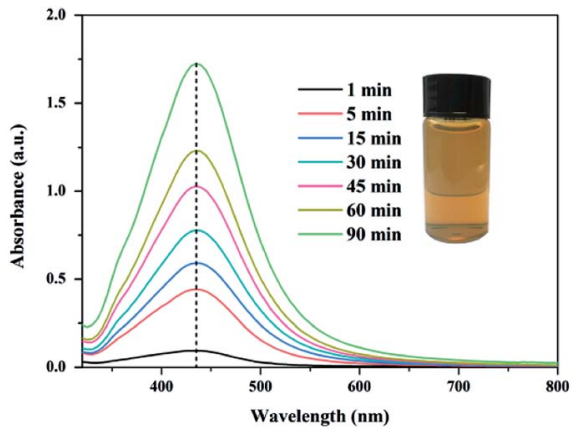

Fig. 2 UV-vis spectra of AgNPs/PVA solution and the insets is the photograph of the corresponding AgNPs solution.

heated for $90 \mathrm{~min}$ at $65{ }^{\circ} \mathrm{C}$, which is also a manifest of characteristic SPR of AgNPs. ${ }^{44,45}$

\subsection{Morphological analyses of nanofibres}

Fig. 3 shows the typical SEM picture of neat PVA, PVA/AgNPs, neat PCL, and PCL/AP. The formation of AgNPs and the addition of AP attributed to the increasing of electrospun solutions conductivity. The higher conductivity leads to larger residual
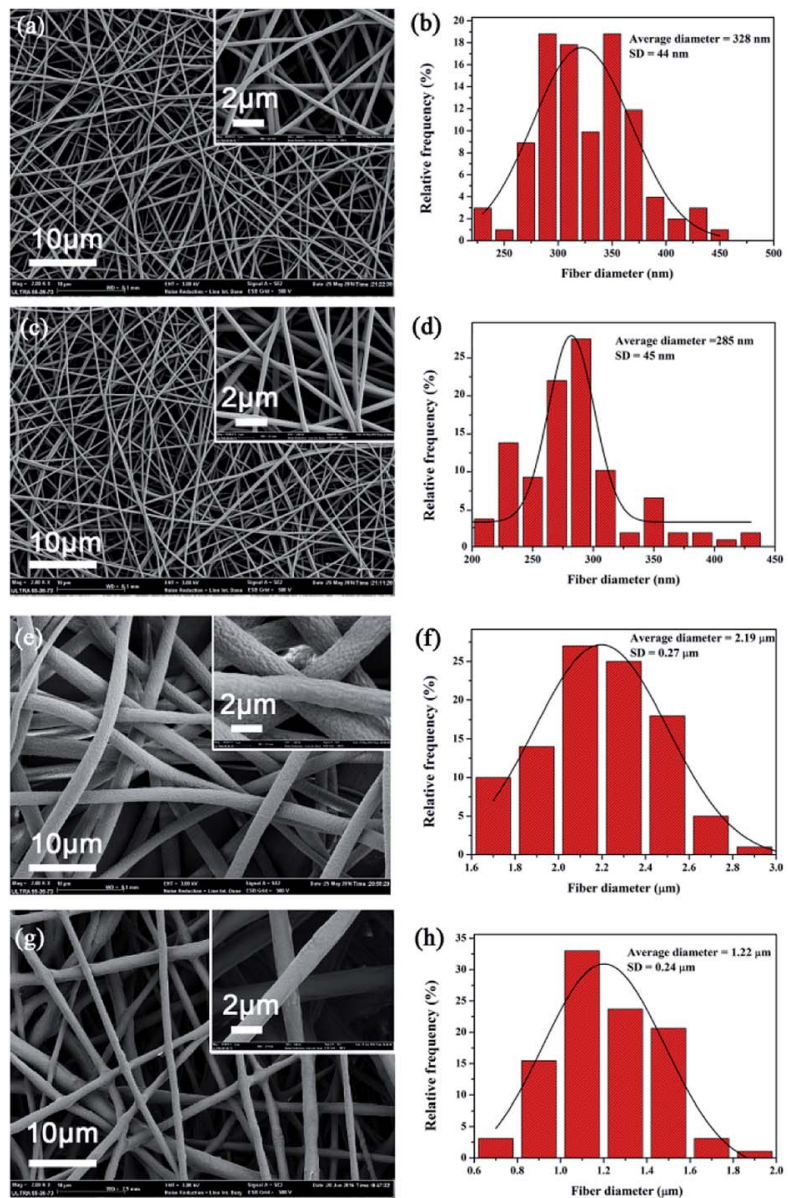

Fig. 3 Typical SEM images of nanofibrous mats: (a) neat PVA, (c) PVA/ AgNPs, (e) neat PCL, (g) PCL/AP, and (b), (d), (f), (h) the statistic frequency distribution of obtained (a), (c), (e) and (g).
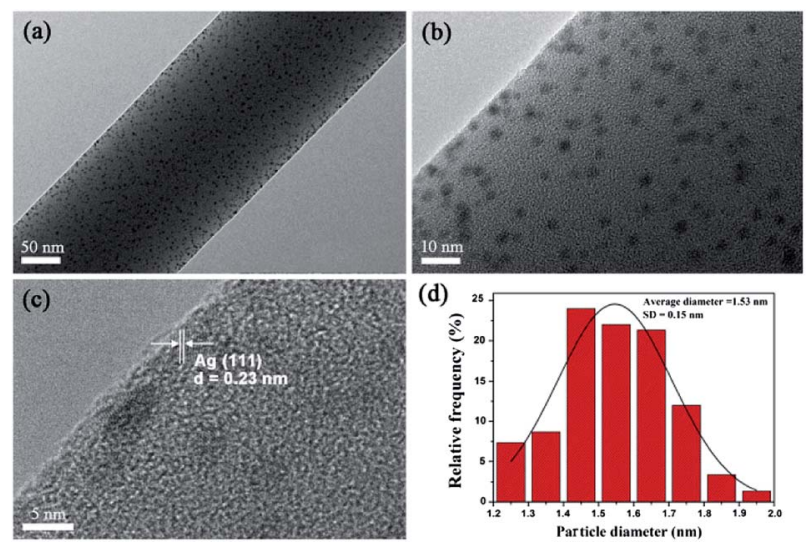

Fig. 4 ( $a$ and b) TEM observation of the PVA/AgNPs, (c) HRTEM image of AgNPs (inter-plane spacing $c$ is $0.23 \mathrm{~nm}$ ), and (d) histograms of AgNPs diameter distribution.

positive charges loading on the jet surface, which directly causes a greater electrostatic repulsion, and thus fibres are stretched into thinner fibers with a smaller diameter. The average diameter of PVA/AgNPs (i.e. $285 \pm 45 \mathrm{~nm}$ ) is smaller than the neat PVA (i.e. $328 \pm 44 \mathrm{~nm}$ ), and the average diameter of PCL/AP (i.e. $1.22 \pm 0.24 \mu \mathrm{m}$ ) is nearly half of the neat PCL (i.e. $2.19 \pm 0.27 \mu \mathrm{m})$.

In TEM images (see Fig. 4), uniformly dispersed AgNPs with a mean diameter of $1.53 \pm 0.15 \mathrm{~nm}$ embedded in electrospun PVA nanofibres. HRTEM image shows the lattice fringes with a $d$-spacing of $0.23 \mathrm{~nm}$ corresponding to the lattice spacing of the (1 111 ) planes of the $\mathrm{Ag}$ face-centered cubic (fcc) facet. ${ }^{23}$

Fig. 5 summarizes the typical reduction reaction procedure for the preparation of AgNPs/PVA nanofibres. The synthesis of AgNPs of different sizes was a kinetically driven process. The PVA possesses a large amount of hydroxyl groups, which could effectively catch $\mathrm{Ag}^{+}$ions in the PVA solution through chelating effect. With the adding of the TP, the phenolic hydroxyls were introduced into the reaction. The $\mathrm{Ag}^{+}$ions were in situ-reduced to AgNPs by phenolic hydroxyls. Also, in the high viscosity PVA solution, the diffusion of the $\mathrm{Ag}^{+}$ions was slowed down, thereby leading to the nucleus growing at a relatively low rate. The

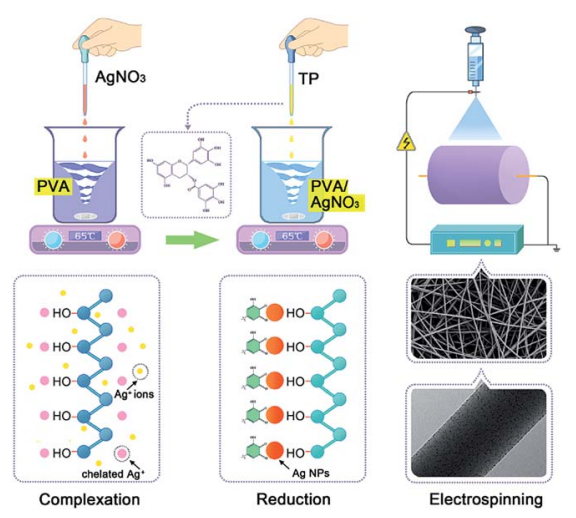

Fig. 5 A schematic illustration of the mechanism for the fabrication of PVA/AgNPs nanofibres. 

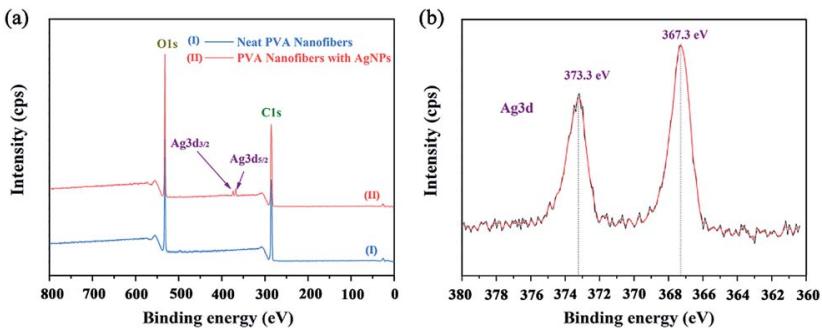

Fig. 6 XPS spectra of AgNPs/PVA hybrids. (a) Survey scan spectrum. (b) High-resolution spectrum for Ag 3d region.
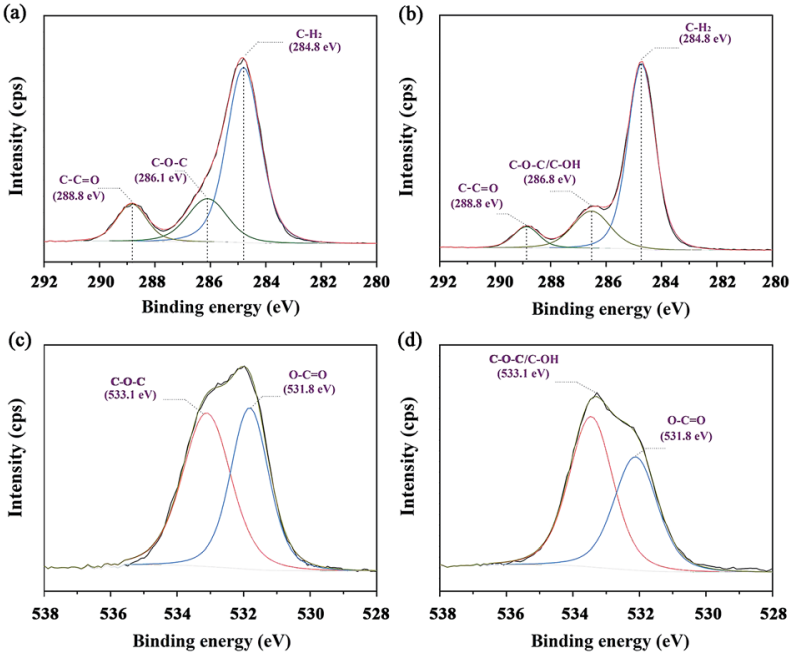

Fig. 7 Narrow scans of $C 1$ s and $O 1$ s spectra of (a and $c$ ) neat PCL and (b and d) PCL/AP, and their fitting curves.

chelating effect between the $\mathrm{Ag}^{+}$ions and hydroxyl groups in PVA, and high viscosity of the PVA solution may play an important role restraining the free growth and aggregation of AgNPs. ${ }^{46}$

\subsection{Further characterization}

Further XPS analysis confirms the presence of $\mathrm{Ag}$ in the composites, and the typical XPS spectra are presented in Fig. 6. The neat PVA only showed $\mathrm{C}$ and $\mathrm{O}$ signals (Fig. 6a, line I), whereas PVA/Ag had C, O, and Ag signals (Fig. 6a, line II). Fig. 6b shows the XPS spectrum in the Ag $3 \mathrm{~d}$ region of the AgNPs/PVA nanofibres. It can be seen from the spectrum that the two XPS peaks at $373.3 \mathrm{eV}$ and $367.3 \mathrm{eV}$ were in agreement the binding energies of $\mathrm{Ag} 3 \mathrm{~d}_{5 / 2}$ and $\mathrm{Ag} 3 \mathrm{~d}_{3 / 2}$, respectively, in accordance with the zero valence of $\mathrm{Ag}$ atom. ${ }^{47}$

The XPS spectra of the $\mathrm{C} 1 \mathrm{~s}$ in neat PCL and PCL/AP nanofibres are shown in Fig. 7a and b. In neat PCL, the carbon atoms participating in the $\mathrm{C}-\mathrm{C}=\mathrm{O}$ is almost equal to the one in the $\mathrm{C}-\mathrm{O}-$ C. Meanwhile, in PCL/AP nanofibres, the characteristic peak at $286.8 \mathrm{eV}$ was higher than the peak at $288.8 \mathrm{eV}$, suggesting the increase in the atomic percentages of the carbon atoms participating in $\mathrm{C}-\mathrm{O}-\mathrm{C} / \mathrm{C}-\mathrm{OH}$ of the vitamin structure. Likewise, the same result confirmed by the recorded $\mathrm{O} 1 \mathrm{~s}$ spectra, and the typical XPS spectra are presented in Fig. $7 \mathrm{c}$ and d. With the adding of the AP in the PCL nanofibres, there is a certain increase in the peak area of the O-atoms, participating in $\mathrm{C}-\mathrm{O}-\mathrm{C} / \mathrm{C}-\mathrm{OH}$ bonds. ${ }^{27}$

The crystalline structure of the nanofibres was determined by XRD. Fig. 8a shows two distinct characteristic peaks in the PVA/AgNPs mat at the $2 \theta$ values of $38.0^{\circ}$, and $77.3^{\circ}$, corre-

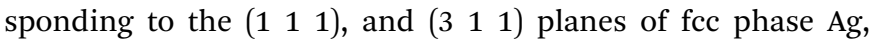
respectively (JCPDS Card No. 04-0783). ${ }^{48}$ In the PCL/AP, a weak diffraction peak located at $15^{\circ}$, can be attributed to the characteristic peak of the AP (see Fig. 8b). The PVA/AgNPs/PCL/AP exhibits the mixed pattern of PVA/AgNPs and PCL/AP. Or to be specific, each component would show its own crystal region in the hybrid nanofibres, and the typical XRD patterns are presented in Fig. 8c.

The characteristic group changes of the nanofibres mats were examined by FTIR spectrum. Neat PVA and PVA/AgNPs have the same peaks, indicating that the adding of AgNPs no effect on the chemical group of the PVA nanofibres (see Fig. 9a). The FTIR spectrum of the PCL/AP (see Fig. 9b) contained a peak at $1660 \mathrm{~cm}^{-1}$, which is the stretch vibration of $\mathrm{AP} \mathrm{C}=\mathrm{C}$ group. Meanwhile, in the PVA/AgNPs/PCL/AP nanofibres, the absorption peaks at $3307 \mathrm{~cm}^{-1}$ (stretch vibration $\mathrm{O}-\mathrm{H}$ group) and 1723 $\mathrm{cm}^{-1}$ (stretch vibration of $\mathrm{C}=\mathrm{O}$ group) are characteristic to PVA and PCL (see Fig. 9c). This result also indicated that the hybrid mat successfully combines of PVA/AgNPs and PCL/AP.

\subsection{Antimicrobial activity}

Preventing the bacterial infection has the prime importance in the inflammatory phase. Fig. 10 shows the antibacterial activity
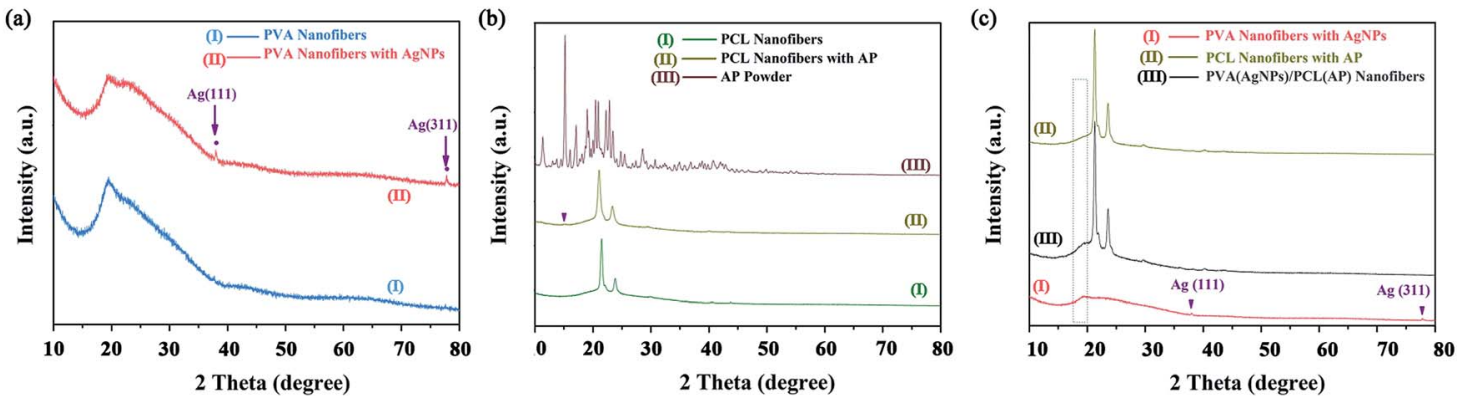

Fig. 8 The XRD pattern of the nanofibres mats: (a) neat PVA and PVA/AgNPs, (b) neat PCL and PCL/AP, and (c) PVA/AgNPs, PCL/AP and hybrid PVA/AgNPs/PCL/AP. 
(a)

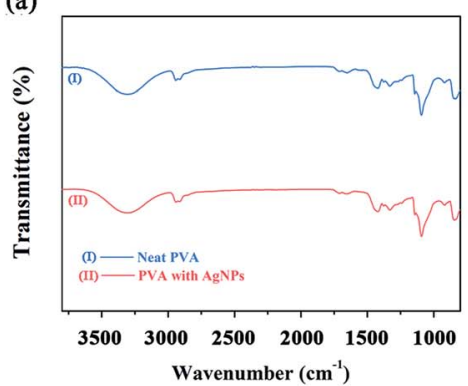

(b)

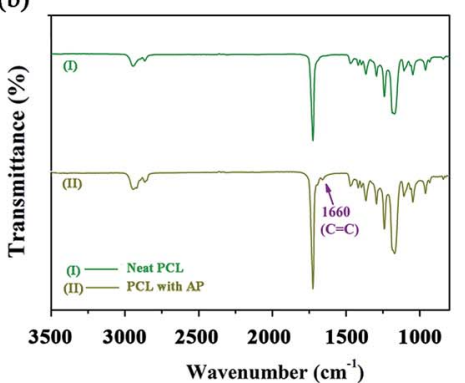

(c)

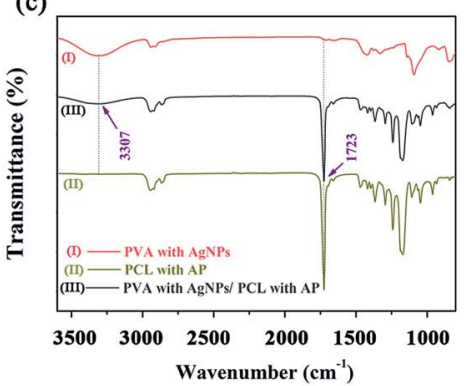

Fig. 9 FTIR spectra of the nanofibres mats: (a) neat PVA and PVA/AgNPs, (b) neat PCL and PCL/AP, and (c) PVA/AgNPs, PCL/AP and hybrid PVA/ AgNPs/PCL/AP.

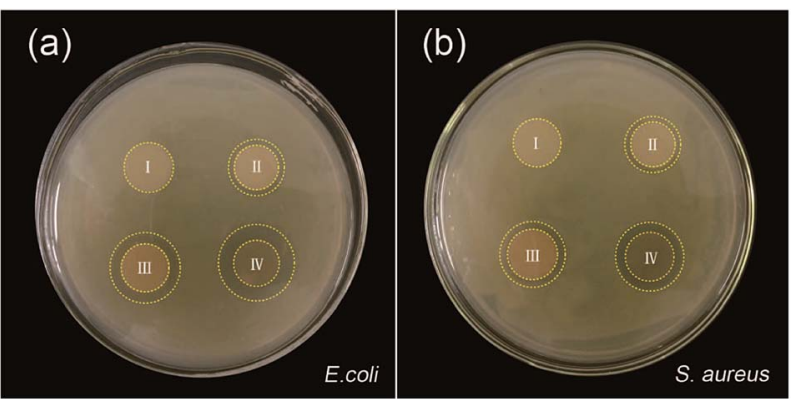

Fig. 10 The antibacterial activity of hybrid nanofibres mats in the presence of (a) E. coli, and (b) S. aureus.

of the electrospun nanofibre mats through a zone inhibition test. The neat PVA/PCL nanofibres mats do not inhibit $E$. coli and $S$. aureus growth at all. The hybrid nanofibrous mats with AP exhibits a good antibacterial effect. In comparison with the hybrid nanofibrous mats with AP, the other samples with AgNPs demonstrate stronger antibacterial activity. $\mathrm{Ag}^{+}$can bind to the thiol groups $(-\mathrm{SH})$ in enzymes, causing cellular enzymes structural which prevents cell division and DNA replication. ${ }^{49-51}$ AgNPs may serve as a vehicle to deliver $\mathrm{Ag}^{+}$more effectively to the bacteria cytoplasm and membrane (Fig. 11). ${ }^{52}$ At the same time, the antibacterial activity is closely related to the size of nanoparticles. The nanoparticles mainly in the range of 1-10 $\mathrm{nm}$ can present a direct interaction with the bacteria preferentially (being less susceptible to binding and reduced bioavailability by common natural ligands). ${ }^{53,54}$ The hybrid

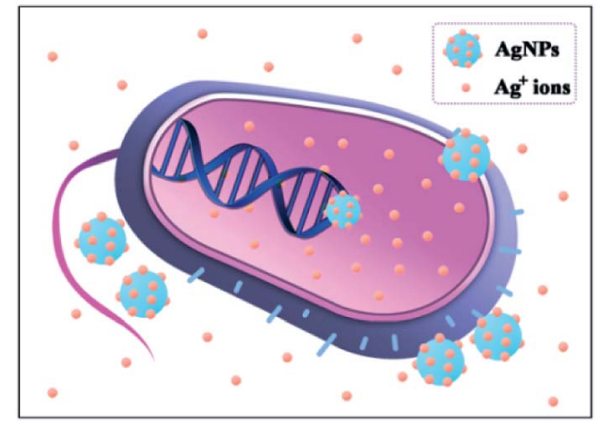

Fig. 11 Schematic of AgNPs and $\mathrm{Ag}^{+}$ions interact with bacteria. nanofibres mats with AgNPs and AP shows strongest antimicrobial property, because of the combined action of AgNPs and AP. Moreover, the Gram-negative bacteria E. coli shows wider zone inhibition than that of Gram-positive bacteria $S$. aureus, which may since the Gram-positive bacteria are protected by a thicker peptidoglycan wall that limits the penetration of the antibacterial agent. ${ }^{55}$ The diameter of inhibition zones around the disc containing silver nanoparticles in E. coli and $S$. aureus are provided in Table 1.

\subsection{Cytotoxicity of electrospun hybrid nanofibres}

An ideal wound dressing should be nontoxic to human tissue. To evaluate the cytotoxicity of the prepared nanofibres mats,

Table 1 Diameter of inhibitory zone by agar diffusion method using composite mats on E. coli and S. aureus

\begin{tabular}{lll}
\hline & \multicolumn{2}{l}{ Inhibitory zone $(\mathrm{mm})$} \\
\cline { 2 - 3 } Sample & E. coli & S. aureus \\
\hline Neat PVA/PCL & 0 & 0 \\
PVA/PCL/AP & $1.4 \pm 0.3$ & $1.3 \pm 0.2$ \\
PVA/PCL/AgNPs & $2.6 \pm 0.5$ & $2.1 \pm 0.3$ \\
PVA/PCL/AgNPs/AP & $3.6 \pm 0.4$ & $3.2 \pm 0.3$
\end{tabular}

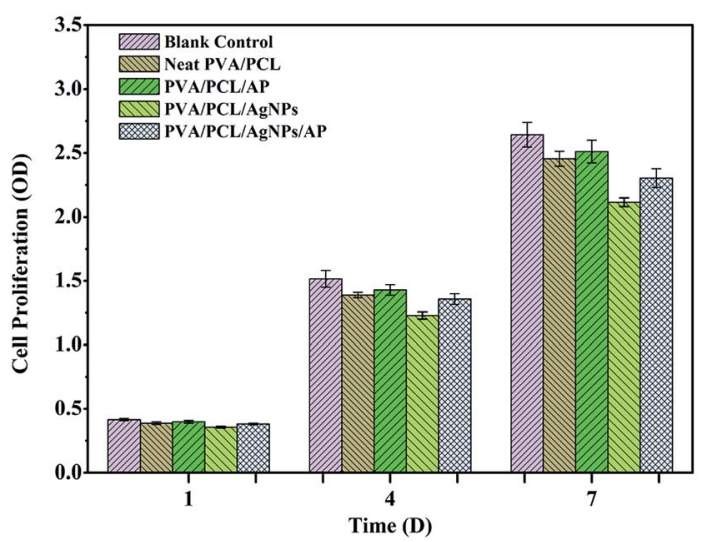

Fig. 12 Cytotoxicity studies using MTT assay after culturing the NIH$3 T 3$ fibroblasts for 1,4 , and 7 days. 
nanofibres were seeded with $\mathrm{NIH}-3 \mathrm{~T} 3$ fibroblasts and results of cell viability is shown in Fig. 12. No remarkable differences were observed in the cell viability of $\mathrm{NIH}-3 \mathrm{~T} 3$ fibroblasts for one day in all of the samples. In comparison with the control sample, the absorbance values of nanofibres mats are lower than that of the control condition on the seventh day. The nanofibres mats

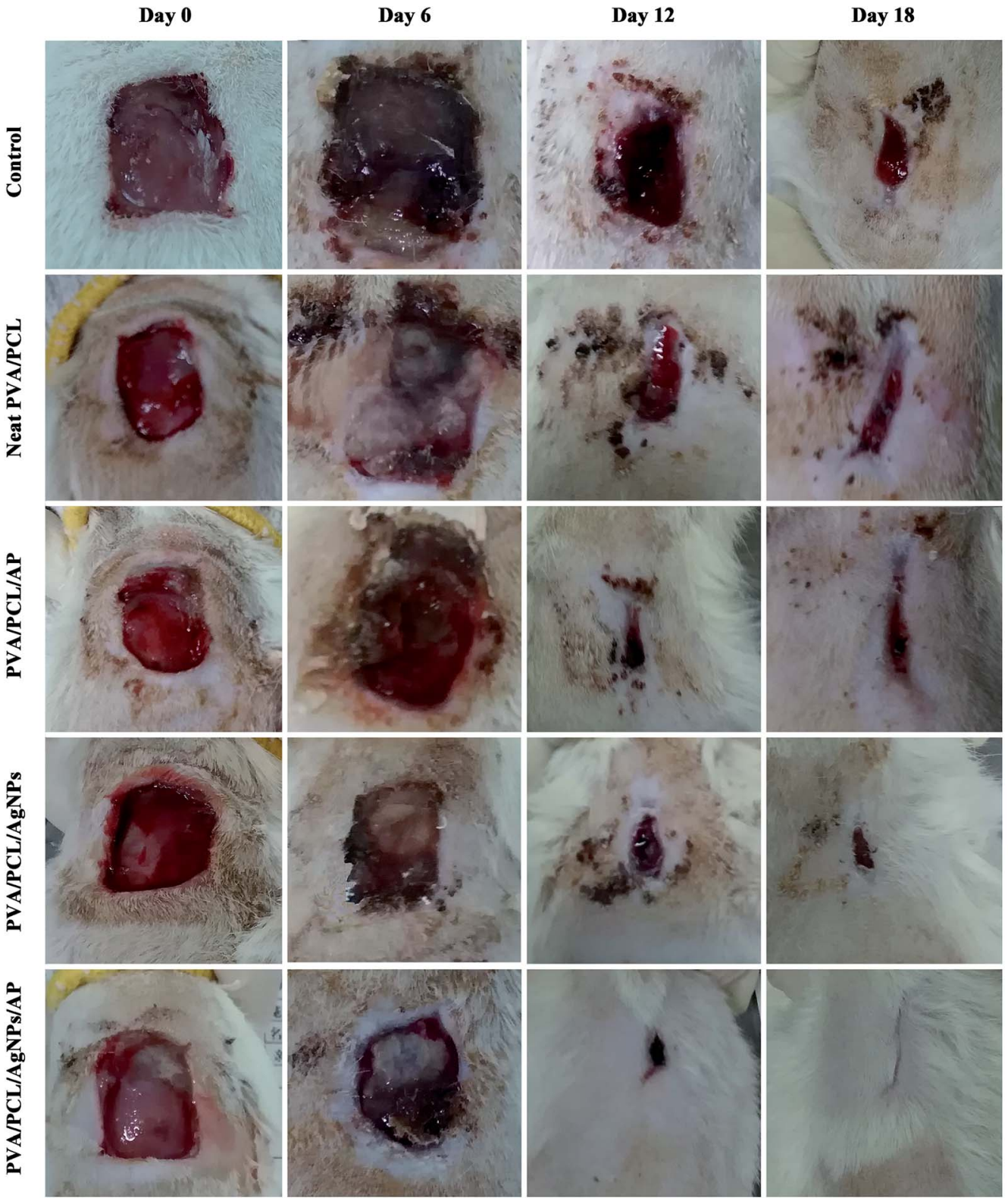

(b)

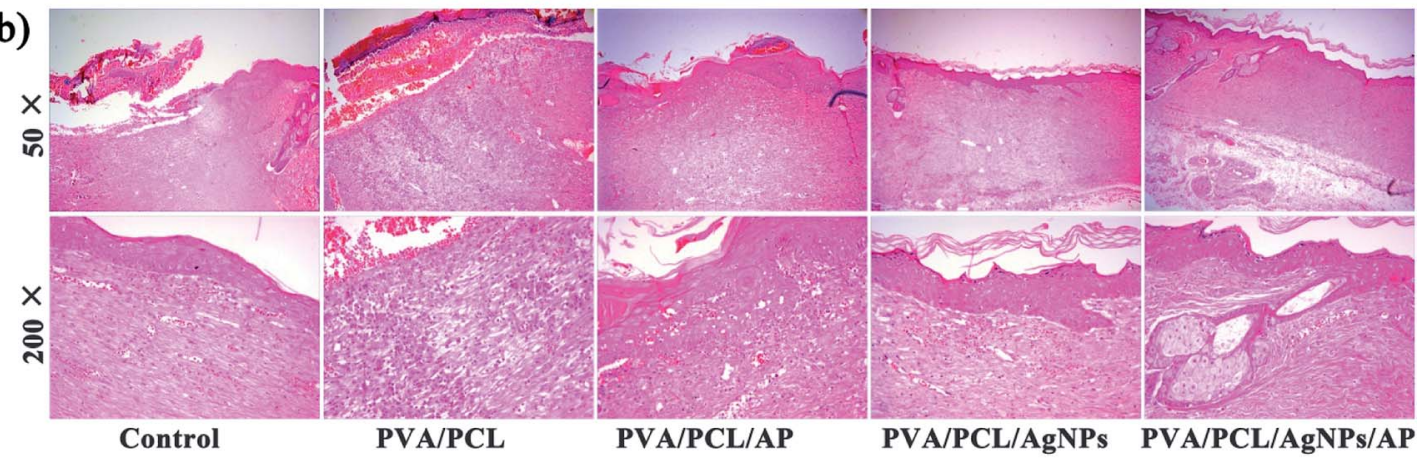

Fig. 13 (a) The wound appearance observed at designed time points. (b) Photomicrographs of hematoxylin and eosin (H\&E)-stained. 
only embedded AgNPs displays a corresponding decrease in cell viability which implies that the loading of AgNPs affect the biocompatibility of nanofibre mats. The rationale may be that AgNPs released the free silver ions which contribute to the toxicity in $\mathrm{NIH}-3 \mathrm{T3}$ fibroblasts. ${ }^{56,57}$ Surprisingly, fibroblast cells proliferated more in the nanofibres mats with AP as compared to without one. Meanwhile, the absorbance values of hybrid nanofibres mat with AgNPs and AP is above $87 \%$ compared with that of the control. The results indicate that hybrid nanofibres mat with AgNPs and AP is not obviously toxic to $\mathrm{NIH}-3 \mathrm{T3}$ fibroblasts and support cell proliferation.

\subsection{Wound closure analysis}

After the antibacterial test and the evaluation of the biocompatibility, the ability of the nanofibres mats to aid wound healing in vivo was explored by creating full-thickness skin wounds on the back of rats. The appearance of wounds after treatment with different dressings is shown in Fig. 13a. Each wound was studied for a period of 6,12 and 18 days post-operation. In all groups, the rats all were vivid and did not show skin-sensitive side effects. At 6th day, wound closure in the treatment group was similar to the control group. Although all the wound size tends to decrease at 12th and 18th day, there are remarkable differences between five groups. By day 18, the healing in the treatment groups was visibly faster than those in the control group. The wounds of the rats treated with PVA/PCL/AgNPs/AP were almost invisible since no redness could be seen and minimal scarring had occurred. At the same time, the rats of other groups still showed visible scars. The PVA/PCL/AgNPs/AP showed a highest wound-healing ratio of 99\%. The wound-healing ratio of the PVA/PCL/AgNPs, PVA/PCL/ AP, neat PVA/PCL, and control group were 94\%, 91\%, 85\% and $77 \%$, respectively. Best results with AgNPs and AP composite PVA/PCL nanofibres mats can be credited to the fact that the antimicrobial hybrid provided an anti-inflammatory environment resulting in reduced reactive cell infiltration, which further aided in the deposition of the collagen to accelerate the formation and engraftment of the tissue. Or to be specific, the AgNPs provided the antibacterial effect; while the AP promoted growth and migration of cells, which altogether contributed to wound healing. Besides that the histopathological observations of the excision wound tissues of the rats also confirmed the hybrid nanofibres mats accelerate the healing of the wounds as compared to the bare wound (see Fig. 13b). In the control group, lots of inflammatory cells in the dermis and subcutaneous tissue were covered by shattered and incomplete epithelium, which was considered as an indicator of the ongoing process of reepithelization. In comparison, evenly distributed blood vessels were found in the group of PVA/PCL/AP. Sufficient angiogenesis associated with appropriate collagen deposition is necessary for granulation tissue formation, as it fills out defects and prepares the way for epithelialization. ${ }^{58}$ The wound treated with PVA/PCL/ AgNPs showed a complete thickened epidermis, reticular fiber, and granulated tissue formation. In contrast, the number of inflammatory cells decreased markedly in groups treated with hybrid PVA/PCL/AgNPs/AP nanofibers mats. Notably, the complete glandular cavity and blood vessels can easily be observed in this case. All these studies indicated that this advanced PVA/PCL/AgNPs/AP hybrid wound dressings induced better wound healing than the other groups.

\section{Conclusions}

In summary, with dual-spinneret electrospinning, it succeeds in fabricating the AP loaded PCL and AgNPs embedded PVA hybrid mats for using as a wound dressing. The morphology analysis indicates PVA and PCL nanofibres are woven together. The uniform distribution of ultra-small AgNPs (i.e. $1.53 \pm 0.15 \mathrm{~nm}$ ) along the PVA nanofibres indicated the chelating effect between the $\mathrm{Ag}^{+}$ions and hydroxyl groups in PVA and high viscosity of the PVA solution restrain the free growth and aggregation of AgNPs. The presence of AgNPs and AP were confirmed via XPS, $\mathrm{XRD}$, and FTIR. Also, the XRD and FTIR indicated good mixture of PCL/AP and PVA/AgNPs nanofibres in the mats via such dualspinneret technique. The prepared hybrid nanofibre mats showed excellent antibacterial activity against two types of bacterial E. coli and $S$. aureus as Gram-positive and Gramnegative bacterial, respectively. The cytotoxicity of the hybrid nanofibre mats was revealed by using mouse fibroblast cell line (NIH-3T3) and showed that there was not obvious toxicity of the prepared hybrid wound dressing. Moreover, using a validated in vivo animal experiment, it was proved that the hybrid PCL/AP and PVA/AgNPs nanofibres mat could accelerate wound healing compared with other samples. Taken together, our study provides evidences that such hybrid mats with a good biological functionality can be utilized as an ideal wound dressing. More interestingly, the present work might open new doors for the exploration of loading drugs no matter water soluble or fat soluble in hybrid nanofibres.

\section{Acknowledgements}

This work is funded by the Zhejiang top priority discipline of textile science and engineering of the outstanding postgraduate dissertation growth foundation (2014YBPY07).

\section{References}

1 A. GhavamiNejad, A. R. Unnithan, A. R. K. Sasikala, M. Samarikhalaj, R. G. Thomas, Y. Y. Jeong, S. Nasseri, P. Murugesan, D. M. Wu, C. H. Park and C. S. Kim, ACS Appl. Mater. Interfaces, 2015, 7, 12176-12183.

2 J. Boateng and O. Catanzano, J. Pharm. Sci., 2015, 104, 36533680 .

3 K. Kataria, A. Gupta, G. Rath, R. B. Mathur and S. R. Dhakate, Int. J. Pharm., 2014, 469, 102-110.

4 R. Agarwal, M. S. Alam and B. Gupta, J. Appl. Polym. Sci., 2013, 129, 3728-3736.

5 N. Monteiro, M. Martins, A. Martins, N. A. Fonseca, J. N. Moreira, R. L. Reis and N. M. Neves, Acta Biomater., 2015, 18, 196-205.

6 A. H. Wang, C. Xu, C. W. Zhang, Y. N. Gan and B. Wang, J. Nanomater., 2015, 2015, 1-8. 
7 R. H. Dong, Y. X. Jia, C. C. Qin, L. Zhan, X. Yan, L. Cui, Y. Zhou, X. Jiang and Y. Z. Long, Nanoscale, 2016, 8, 34823488.

8 W. A. Sarhan, H. M. E. Azzazy and I. M. El-Sherbiny, ACS Appl. Mater. Interfaces, 2016, 8, 6379-6390.

9 J. Chutipakdeevong, U. Ruktanonchai and P. Supaphol, J. Appl. Polym. Sci., 2014, 132, 41653-41663.

10 F. Cheng, J. Gao, L. Wang and X. Y. Hu, J. Appl. Polym. Sci., 2015, 132, 42060-42067.

11 Y. Gao, Y. B. Truong, Y. G. Zhu and I. L. Kyratzis, J. Appl. Polym. Sci., 2014, 131, 40797-40809.

12 P. I. Morgado, A. Aguiar-Ricardo and I. J. Correia, J. Membr. Sci., 2015, 490, 139-151.

13 P. Dubey, B. Bhushan, A. Sachdev, I. Matai, S. U. Kumar and P. Gopinath, J. Appl. Polym. Sci., 2015, 132, 42473-42484.

14 Y. Wan, Z. R. Guo, X. L. Jiang, K. Fang, X. Lu, Y. Zhang and N. Gu, J. Colloid Interface Sci., 2013, 394, 263-269.

15 H. Y. Son, J. H. Ryu, H. Lee and Y. S. Nam, Macromol. Mater. Eng., 2013, 298, 547-554.

16 A. M. Abdelgawad, S. M. Hudson and O. J. Rojas, Carbohydr. Polym., 2014, 100, 166-178.

17 M. S. Islam and M. R. Karim, Colloids Surf., A, 2010, 366, 135140.

18 Z. Q. Cheng, F. L. Zhang, W. Liu, L. Y. Cui and L. J. Kang, RSC Adv., 2015, 5, 54182-54187.

19 W. J. Jin, H. J. Jeon, J. H. Kim and J. H. Youk, Synth. Met., 2007, 157, 454-459.

20 S. Lin, R. Z. Wang, Y. Yi, Z. Wang, L. M. Hao, J. H. Wu, G. H. Hu and H. He, Int. J. Nanomed., 2014, 9, 3937-3947.

21 A. K. Selvam and G. Nallathambi, Fibers Polym., 2015, 16, 1327-1335.

22 V. K. Shukla, R. P. Singh and A. C. Pandey, J. Alloys Compd., 2010, 507, L13-L16.

23 H. Zhu, M. L. Du, M. Zhang, P. Wang, S. Y. Bao, Y. Q. Fu and J. M. Yao, Sens. Actuators, B, 2013, 185, 608-619.

24 H. Zhu, M. L. Du, M. Zhang, P. Wang, S. Y. Bao, Y. Q. Fu and J. M. Yao, Biosens. Bioelectron., 2013, 49, 210-215.

25 M. L. Zou, M. L. Du, H. Zhu, C. S. Xu, N. Li and Y. Q. Fu, Polym. Eng. Sci., 2013, 53, 1099-1108.

26 J. S. Boateng, K. H. Matthews, H. N. Stevens and G. M. Eccleston, J. Pharm. Sci., 2008, 97, 2892-2923.

27 D. Paneva, N. Manolova, M. Argirova and I. Rashkov, Int. J. Pharm., 2011, 416, 346-355.

28 R. Yoksan, J. Jirawutthiwongchai and K. Arpo, Colloids Surf., $B, 2010,76,292-297$.

29 N. S. Barbosa and A. N. Kalaaji, Compl. Ther. Clin. Pract., 2014, 20, 11-15.

30 S. Sridhar, J. R. Venugopal and S. Ramakrishna, J. Biomed. Mater. Res., Part A, 2015, 103, 3431-3440.

31 M. Abrigo, S. L. McArthur and P. Kingshott, Macromol. Biosci., 2014, 14, 772-792.

32 R. Z. Wang, Z. Wang, S. Lin, C. Deng, F. Li, Z. J. Chen and H. He, RSC Adv., 2015, 5, 40141-40147.

33 L. D. Tijing, M. T. G. Ruelo, A. Amarjargal, H. R. Pant, C. H. Park and C. S. Kim, Mater. Chem. Phys., 2012, 134, $557-561$.
34 C. Q. Zhang, Y. P. Li, W. Wang, N. Q. Zhan, N. Xiao, S. Wang, Y. X. Li and Q. B. Yang, Eur. Polym. J., 2011, 47, 2228-2233.

35 R. Zhao, X. Li, B. L. Sun, Y. Tong, Z. Q. Jiang and C. Wang, $R S C$ Adv., 2015, 5, 16940-16949.

36 A. Baranowska-Korczyc, A. Warowicka, M. JasiurkowskaDelaporte, B. Grześkowiak, M. Jarek, B. M. Maciejewska, J. Jurga-Stopa and S. Jurga, RSC Adv., 2016, 6, 19647-19656.

37 N. B. Halima, RSC Adv., 2016, 6, 39823-39832.

38 T. Kaur and A. Thirugnanam, RSC Adv., 2016, 6, 3998239992.

39 M. Koosha, H. Mirzadeh, M. A. Shokrgozar and M. Farokhi, RSC Adv., 2015, 5, 10479-10487.

40 N. Manhas, K. Balasubramanian, P. Prajith, P. Rule and S. Nimje, RSC Adv., 2015, 5, 23999-24008.

41 E. J. Chong, T. T. Phan, I. J. Lim, Y. Z. Zhang, B. H. Bay, S. Ramakrishna and C. T. Lim, Acta Biomater., 2007, 3, 321-330.

42 Q. L. Wei, F. Y. Xu, X. J. Xu, X. Geng, L. Ye, A. Y. Zhang and Z. G. Feng, Front. Mater. Sci., 2016, 10, 113-121.

43 A. Chaturvedi, A. K. Bajpai, J. Bajpai and K. S. Singh, Mater. Sci. Eng., C, 2016, 65, 408-418.

44 V. K. Sharma, R. A. Yngard and Y. Lin, Adv. Colloid Interface Sci., 2009, 145, 83-96.

45 M. C. Moulton, L. K. Braydich-Stolle, M. N. Nadagouda, S. Kunzelman, S. M. Hussain and R. S. Varma, Nanoscale, 2010, 2, 763-770.

46 P. Wang, H. Zhu, S. Y. Bao, M. L. Du and M. Zhang, J. Phys. D: Appl. Phys., 2013, 46, 345303-345310.

47 J. Zhang, Y. Yuan, X. W. Xu, X. L. Wang and X. R. Yang, ACS Appl. Mater. Interfaces, 2011, 3, 4092-4100.

48 Z. L. Xu, K. Miyazaki and T. Hori, Appl. Surf. Sci., 2016, 370, 243-251.

49 M. K. Rai, S. D. Deshmukh, A. P. Ingle and A. K. Gade, J. Appl. Microbiol., 2012, 112, 841-852.

50 Q. L. Feng, J. Wu, G. Q. Chen, F. Z. Cui, T. N. Kim and J. O. Kim, J. Biomed. Mater. Res., Part A, 2010, 52, 662-668.

51 G. Franci, A. Falanga, S. Galdiero, L. Palomba, M. Rai, G. Morelli and M. Galdiero, Molecules, 2015, 20, 8856-8874.

52 Z. M. Xiu, J. Ma and P. J. J. Alvarez, Environ. Sci. Technol., 2011, 45, 9003-9008.

53 J. R. Morones, J. L. Elechiguerra, A. Camacho, K. Holt, J. B. Kouri, J. T. Ramirez and M. J. Yacaman, Nanotechnology, 2005, 16, 2346-2353.

54 H. M. Braakhuis, I. Gosens, P. Krystek, J. A. Boere, F. R. Cassee, P. H. Fokkens, J. A. Post, H. van Loveren and M. V. Park, Part. Fibre Toxicol., 2014, 11, 49-64.

55 J. S. Kim, E. Kuk, K. N. Yu, J. H. Kim, S. J. Park, H. J. Lee, S. H. Kim, Y. K. Park, Y. H. Park, C. Y. Hwang, Y. K. Kim, Y. S. Lee, D. H. Jeong and M. H. Cho, Nanomedicine, 2007, 3, 95-101.

56 R. P. Singh and P. Ramarao, Toxicol. Lett., 2012, 213, 249259.

57 C. Beer, R. Foldbjerg, Y. Hayashi, D. S. Sutherland and H. Autrup, Toxicol. Lett., 2012, 208, 286-292.

58 H. Niiyama and Y. Kuroyanagi, J. Artif. Organs, 2014, 17, 8187. 